\title{
Optimal control for colistin dosage selection
}

\author{
Aline Vidal Lacerda Gontijo ${ }^{1,2}$ (1) $\cdot$ André V. G. Cavalieri ${ }^{3}$ (i)
}

Received: 3 April 2021 / Accepted: 12 June 2021 / Published online: 22 June 2021

(C) The Author(s), under exclusive licence to Springer Science+Business Media, LLC, part of Springer Nature 2021

\begin{abstract}
Optimization of antibiotic administration helps minimizing cases of bacterial resistance. Dosages are often selected by trial and error using a pharmacokinetic (PK) model. However, this is limited to the range of tested dosages, restraining possible treatment choices, especially for the loading doses. Colistin is a last-resort antibiotic with a narrow therapeutic window; therefore, its administration should avoid subtherapeutic or toxic concentrations. This study formulates an optimal control problem for dosage selection of colistin based on a PK model, minimizing deviations of colistin concentration to a target value and allowing a specific dosage optimization for a given individual. An adjoint model was used to provide the sensitivity of concentration deviations to dose changes. A three-compartment PK model was adopted. The standard deviation between colistin plasma concentrations and a target set at $2 \mathrm{mg} / \mathrm{L}$ was minimized for some chosen treatments and sample patients. Significantly lower deviations from the target concentration are obtained for shorter administration intervals (e.g. every $8 \mathrm{~h}$ ) compared to longer ones (e.g. every $24 \mathrm{~h}$ ). For patients with normal or altered renal function, the optimal loading dose regimen should be divided into two or more administrations to attain the target concentration quickly, with a high first loading dose followed by much lower ones. This regimen is not easily obtained by trial and error, highlighting advantages of the method. The present method is a refined optimization of antibiotic dosage for the treatment of infections. Results for colistin suggest significant improvement in treatment avoiding subtherapeutic or toxic concentrations.
\end{abstract}

Keywords Optimal control $\cdot$ Colistin $\cdot$ PK model $\cdot$ Optimization of drug administration

\section{Introduction}

The treatment of some infections is a real challenge in the clinical area, since certain bacteria have become resistant to most of available antibiotics, and there are only few new drugs being developed against these bacteria [1]. Therefore, the proper use of the remaining antibiotic options is required to avoid these cases, choosing the most suitable one and the optimal regimen of the treatment [2].

Aline Vidal Lacerda Gontijo

aline.gontijo@gmail.com

1 Department of Clinical and Toxicological Analysis, Federal University of Alfenas, Rua Gabriel Monteiro da Silva 700, Centro, Alfenas, MG 37130-001, Brazil

2 Department of Pharmacy, Anhanguera Educacional, São José dos Campos, SP, Brazil

3 Divisão de Engenharia Aeroespacial, Instituto Tecnológico de Aeronáutica, São José dos Campos, SP, Brazil
The minimum inhibitory concentration (MIC) is a relevant pharmacodynamic (PD) parameter $[3,4]$. The consideration of MIC with other pharmacokinetic (PK) parameters, such as area under the curve (AUC), peak plasma concentration $\left(\mathrm{C}_{\max }\right)$ and time period of plasma concentration above the MIC, helps in choosing the proper use of antibiotics [3-5]. However, some antibiotics have a narrow therapeutic window. In these cases, the plasma concentration should be controlled and cannot be several times higher than the MIC [6].

Modern models and simulations integrating PK and PD are being employed in order to predict plasma concentrations and suggest a suitable treatment scheme [7-9]. Usually, a range of doses is tested and the best dose is selected considering the fittest pharmacokinetic profile produced $[10,11]$. Such trial and error procedures have undoubtedly contributed to the appropriate use of antibiotics. However, the dose selection is limited to the range of tested doses, which is often based on an initial loading dose followed by a number of equal maintenance doses. While this could 
lead to satisfactory effects for most drugs, there are some molecules that may require a more refined treatment optimization, especially in cases where there is a narrow therapeutic window and a target concentration should be attained.

Colistin is an old drug which was commercially available in the 1950s but rapidly replaced by other drugs due to its nephrotoxicity $[12,13]$. Nonetheless, cases of resistance to these other drugs emerged and colistin has been shown to be a viable therapeutic option against pathogens which are very difficult to treat, such as Gram-negative bacteria $[14,15]$. For this reason, interest in colistin was renewed in the 2000s, and it has been used as a last resort antibiotic [12]. Intravenous (IV) administration of colistin is often used in cases of nosocomial pneumonia (ventilator-associated or not) due to multidrug-resistant (MDR) or extensively drug-resistant (XDR) pathogens, such as Acinetobacter baumannii, Pseudomonas aeruginosa and Klebsiella pneumoniae [16, 17], since after IV administration, the molecule is able to cross the blood-air barrier and reach the lung tissues $[18,19]$. Colistin is administered as an inactive prodrug, colistimethate sodium (CMS), to mitigate its toxicity [12]. Once administered, CMS is hydrolyzed in colistin, most probably in the bladder of the patients, and approximately $70 \%$ of the CMS dose is rapidly eliminated in the urine $[20,21]$. Therefore, the dose should be adjusted for patients presenting renal problems $[22,23]$.

Although it has been extensively used in the recent decades, the optimal dosing regimen of colistin remains to be determined [21, 24]. A key aspect is that plasma concentrations of colistin below $2 \mathrm{mg} / \mathrm{L}$ are ineffective $[25,26]$, and concentrations above $2.42 \mathrm{mg} / \mathrm{L}$ lead to toxicity issues [27]. Therefore, colistin administration would greatly benefit from an optimization targeting the safe plasma concentration. Target steady-state concentrations may be obtained using standard methods for linear PK modes [28], but the optimization should also involve the loading dose, in order that the target concentration be rapidly attained. Furthermore, the availability of PK models may allow such optimization to be performed for a specific patient, taking into account how PK parameters vary in the population.

The aim of this study is to use a PK model based optimization to assist the selection of the optimal therapeutic scheme of the colistin. Instead of a selection of doses by trial and error, which invariably limits the possible choices, we formulate an optimization problem that leads to an optimal control formulation. Control theory has been used to optimize treatment in problems with available PK/PD models, such as HIV infections [29, 30], cancer therapy [31-35], or to optimize strategies for the prevention of infectious diseases such as cholera [36], Zika virus infection [37] and COVID-19 [38]. For colistin administration, the optimization problem minimizes the deviations between the plasma concentration of colistin and its target value. Additionally, the optimal regimen was individualized according to the creatinine clearance of the patients. While this work focuses on the specific case of colistin, the formulation is general and allows optimization of administration of other drugs whenever a PK model is available.

\section{Methodology}

\section{Definitions}

We consider the three-compartment PK model from [22], in a simpler form for patients that are neither on hemodyalisis, nor on continuous renal replacement treatment. For these conditions, the model is given by

$$
\begin{aligned}
\frac{d C M S c}{d t}= & R-C L D 1 \times\left(\frac{C M S c}{V 1}-\frac{C M S p}{V 2}\right)-C L T_{C M S} \\
& \times \frac{C M S c}{V 1} \\
\frac{d C M S p}{d t}= & C L D 1 \times\left(\frac{C M S c}{V 1}-\frac{C M S p}{V 2}\right) \\
\frac{d C o l i s t i n}{d t}= & C L N R_{C M S} \times \frac{C M S c}{V 1}-C L T_{c} \times \frac{\text { Colistin }}{V 3}
\end{aligned}
$$

The model variables are the mass of CMS in the central compartment $(C M S c)$, the mass of CMS in the peripheral compartment (CMSp) and the mass of colistin in a single colistin compartment (Colistin).V1, V2 and V3 denote volumes of distribution of the CMSc, CMSp and Colistin compartments, respectively, and CLD1 is the distributional clearance between the central and peripheral compartments for CMS. The administration of CMS in the central compartment is given by $R$ in the above model. The variables in the model refer to the total (bound plus unbound) CMS and colistin. Accordingly, in what follows we will deal solely with total concentrations.

$C L T_{C M S}$ and $C L T_{c}$ are, respectively, the total intrinsic clearances for CMS and colistin. The total CMS clearance $\left(C L T_{C M S}\right)$ was modeled as a function of creatinine clearance $(\mathrm{Cr} C L)$ and two effects, a renal dependent component $\left(C L R_{S L O P E}\right)$ and nonrenal clearance $\left(C L N R_{C M S}\right)$, as

$$
C L T_{C M S}=C r C L \times C L R_{S L O P E}+C L N R_{C M S}
$$

Similarly, the colistin clearance $\left(C L T_{C}\right)$ was modeled as a sum of a renally dependent component $\left(C L R C_{S L_{-} P O P}\right)$ and a nonrenally dependent component $\left(C L N R_{C_{-} P O P}\right)$, as

$$
C L T_{c}=C r C L \times C L R C_{S L_{P O P}}+C L N R_{C_{-} P O P}
$$


A schematic pictogram representing the PK model is shown in Fig. 1. The parameter values used in the simulations are detailed in Table 1.

It is convenient to write the system in matrix form. We define a state as a column vector,

$\boldsymbol{x}=\left[\begin{array}{c}C M S c \\ C M S p \\ \text { Colistin }\end{array}\right]$

the system matrix $\boldsymbol{A}$ as

$A=\left[\begin{array}{ccc}-\frac{C L D 1-C L T_{C M S}}{V 1} & \frac{C L D 1}{V 2} & 0 \\ \frac{C L D 1}{V 1} & -\frac{C L D 1}{V 2} & 0 \\ \frac{C L N R_{C M S}}{V 1} & 0 & -\frac{C L T_{c}}{V 3}\end{array}\right]$

and the input matrix $\boldsymbol{B}$ as

$\boldsymbol{B}=\left[\begin{array}{l}1 \\ 0 \\ 0\end{array}\right]$

such that the system becomes simply

$\frac{d \boldsymbol{x}}{d t}=\boldsymbol{A x}+\boldsymbol{B} u$

with a scalar input given by $u(t)=R(t)$, i.e. the infusion rate of CMS.

The quantity of primary interest in the system is the concentration of colistin in its compartment, given by Colistin/V3. This output, defined $y$, may be extracted by defining an output matrix $\mathbf{C}$ given by

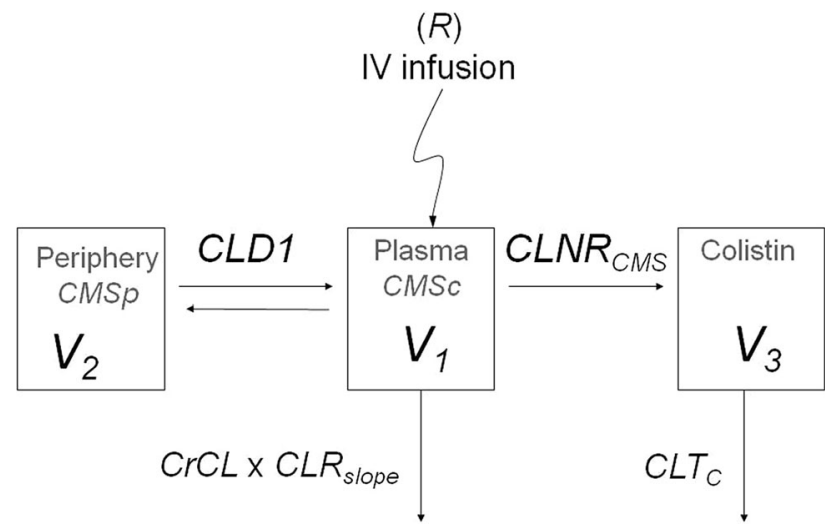

Fig. 1 Structural simultaneous PK modeling: $V 1, V 2$ and $V 3$ volumes of distribution of the central colistimethate sodium $(C M S c)$, peripheral CMS $(C M S p)$ and colistin compartments, respectively, CLD1 distributional clearance between the central and peripheral compartments for CMS, $R$ administration of CMS in the central compartment, $C r C L$ creatinine clearance, $C L R_{S L O P E}$ renal dependent component, $C L N R_{C M S}$ nonrenal clearance, $C L T_{c}$ total intrinsic clearance for colistin
Table 1 PK parameters and covariate values from previous study (22) after IV infusion (R) of CMS in the central compartment used in simulations

\begin{tabular}{lllc}
\hline Category & Parameter & (UNITS) & Value \\
\hline CMS & $\mathrm{V}_{1}$ & $(\mathrm{~L})$ & 11.9 \\
& $\mathrm{~V}_{2}$ & $(\mathrm{~L})$ & 18.7 \\
& CLD1 & $(\mathrm{L} / \mathrm{h})$ & 7.98 \\
& CLR $_{\text {slope }}$ & $(\mathrm{L} / \mathrm{h} / \mathrm{CrCL})$ & 0.0613 \\
& CLNR $_{\mathrm{CMS}}$ & $(\mathrm{L} / \mathrm{h})$ & 1.9 \\
\multirow{4}{*}{ Colistin } & $\mathrm{V}_{3}$ & $(\mathrm{~L})$ & 45.1 \\
& CLT $_{\mathrm{C}}$ & $(\mathrm{L} / \mathrm{h})$ & 2.72 \\
& CLRC $_{\mathrm{SL}_{2} \text { POP }}$ & $(\mathrm{L} / \mathrm{h} / \mathrm{CrCL})$ & 0.0147 \\
& CLNR $_{\mathrm{C}}$ & $(\mathrm{L} / \mathrm{h})$ & 2.19 \\
& Weight & $(\mathrm{Kg})$ & 60 \\
\hline
\end{tabular}

$V 1, V 2$, and $V 3$ volumes of distribution of the central colistimethate sodium (CMS), peripheral CMS and colistin compartments, respectively, $C L D 1$ distributional clearance between the central and peripheral compartments for CMS, $C L R_{S L O P E}$ renal dependent component for CMS, $C L N R_{C M S}$ nonrenal clearance of CMS, $C L T_{c}$ total intrinsic clearance for colistin; $C L R C_{S L_{P} P O P}$ renally dependent component for colistin, $C L N R_{C_{-} P O P}$ nonrenally dependent component for colistin

$\boldsymbol{C}=\left[\begin{array}{lll}0 & 0 & \frac{1}{V 3}\end{array}\right]$

such that

$y=\boldsymbol{C x}$.

The system in Eq. (9). is solved subject to an initial condition, given by $\boldsymbol{x}(t=0)=\boldsymbol{x}_{0}$. Here, the drug administration is assumed to occur after the initial time $t=0$, hence the initial state is given by $\boldsymbol{x}_{0}=0$. Once the solution $\boldsymbol{x}(t)$ is obtained, the colistin concentration is obtained with Eq. (11).

The definition of the system in matrix form is convenient, as will be shown next, and also provides generality. The optimization problem to be defined may be adapted in straigthforward manner to other linear compartmental PK models once they are cast in matrix form by the definitions of system, input and output matrices $\boldsymbol{A}, \boldsymbol{B}$ and $\boldsymbol{C}$, respectively.

\section{Optimization problem}

We define a target concentration $y_{t}(t)$, which may be constant or time-dependent depending on the application. The present optimization problem is set so as to minimize deviations between the model output $y(t)$ and the target concentration. For a target concentration that is constant in time, a maintenance dose may be selected by standard methods for linear PK models [28]. On the other hand, 
obtaining optimal loading doses is not trivial, as it is important to quickly attain the desired concentration for critically ill patients without an overshoot that would possibly be toxic. Selection of does that optimally attain a target concentration that varies with time (i.e. a desired curve of concentration versus time) is also difficult, even for linear models. It is with such purposes that we define the optimization below.

We define the functional $\mathcal{J}$ as

$\mathcal{J}=\frac{1}{2} \int_{0}^{t_{f}}\left[y(t)-y_{t}(t)\right]^{2} d t$

$\mathcal{J}$ is related to the mean square value of the deviations between the colistin concentration and its target value. The quantity $\gamma=\sqrt{2 \mathcal{J} / t_{f}}$ is thus a standard deviation between the colistin concentration and the target, a metric to evaluate if concentrations are close to the target value. We would thus like to obtain the optimal drug administration $u(t)$, such that $\mathcal{J}$ is minimized; a minimisation of $\mathcal{J}$ implies a minimisation of the standard deviation $\gamma$ to the target concentration. Notice that the specification of a target concentration $y_{t}(t)$ is arbitrary. For instance, one may specify a desired concentration curve as a function of time, considered to be ideal for clinical application. The procedure will then determine a drug administration that minimizes deviations to such target curve $y_{t}(t)$.

The present problem is a constrained optimization, where one wishes to minimize the functional $\mathcal{J}$ with respect to $u$ such that the constraints given by Eqs. (9) and (11) are satisfied. This may be solved using Lagrange multipliers or adjoint variables, as in optimal control works dealing with cancer therapy $[32,35]$ or vaccination strategies [37, 38]. The procedure is based on the application of Pontryagin's Maximum Principle [39], which we describe in what follows. We define an augmented functional

$\mathcal{L}(\boldsymbol{x}, \boldsymbol{w}, u)=\frac{1}{2} \int_{0}^{t_{f}}\left(y-y_{t}\right)^{2} d t+\int_{0}^{t_{f}} \boldsymbol{w}^{T}\left(\frac{d \boldsymbol{x}}{d t}-\boldsymbol{A} \boldsymbol{x}-\boldsymbol{B} u\right) d t$

where $\boldsymbol{w}$ is an adjoint variable, introduced to ensure that the constraint is satisfied, simplifying the solution of the problem. Superscript $T$ denotes a matrix transpose. Notice that if $\boldsymbol{x}$ satisfies the PK model of Eq. (9) the last integral is equal to zero, and the augmented functional $\mathcal{L}$ is equal to $\mathcal{J}$. Therefore, minimization of $\mathcal{L}$ will lead to minimisation of $\mathcal{J}$, and, in turn, of the standard deviation $\gamma$.

The unconstrained minimisation of the augmented functional $\mathcal{L}$ is equivalent to the constrained minimisation of $\mathcal{J}$. To show this, consider that once $\mathcal{L}$ is minimized its variation with respect to $\boldsymbol{w}$ should be zero. Denoting such variation by $\delta \mathcal{L}_{\delta w}$, defined by $\delta \mathcal{L}_{\delta \boldsymbol{w}}=\lim _{\delta w \rightarrow 0}[\mathcal{L}(\boldsymbol{x}, \boldsymbol{w}+\delta \boldsymbol{w}, u)-\mathcal{L}(\boldsymbol{x}, \boldsymbol{w}, u)]$

we obtain

$\delta \mathcal{L}_{\delta w}=\int_{0}^{t_{f}} \delta \boldsymbol{w}^{T}\left(\frac{d \boldsymbol{x}}{d t}-\boldsymbol{A x}-\boldsymbol{B} u\right) d t$

where $\delta \boldsymbol{w}$ denotes a small arbitrary variation introduced in $\boldsymbol{w}$. Setting $\delta \mathcal{L}_{w}=0$ for any $\delta \boldsymbol{w}$ implies that

$\frac{d \boldsymbol{x}}{d t}-\boldsymbol{A x}-\boldsymbol{B} u=0$

which means that $\boldsymbol{x}$ is solution to the model in Eq. (9), and the constraint is satisfied.

We now consider the variation of $\mathcal{L}$ with respect to the state $\boldsymbol{x}$, given as

$\delta \mathcal{L}_{\delta x}=\int_{0}^{t_{f}}\left(y-y_{t}\right) \boldsymbol{C} \delta \boldsymbol{x} d t+\int_{0}^{t_{f}} \boldsymbol{w}^{T}\left(\frac{d \delta x}{d t}-\boldsymbol{A} \delta \boldsymbol{x}\right) d t$.

An integration by parts can be applied to the time derivative of $\boldsymbol{x}$, leading to

$$
\begin{aligned}
\delta \mathcal{L}_{\delta \boldsymbol{x}}= & \int_{0}^{t_{f}}\left(y-y_{t}\right) \boldsymbol{C} \delta \boldsymbol{x} d t+\left(\boldsymbol{w}\left(t_{f}\right) \delta \boldsymbol{x}\left(t_{f}\right)-\boldsymbol{w}(0) \delta \boldsymbol{x}(0)\right) \\
& -\int_{0}^{t_{f}} \frac{d \boldsymbol{w}^{T}}{d t} \delta \boldsymbol{x} d t-\int_{0}^{t_{f}} \boldsymbol{w}^{T} \boldsymbol{A} \delta \boldsymbol{x} d t
\end{aligned}
$$

For arbitrary $\delta \boldsymbol{x}$, this variation can be set to zero by the definition of an adjoint system. We first consider that the initial condition for $\boldsymbol{x}$ is fixed, which implies $\delta \boldsymbol{x}(0)=0$. We also specify that the adjoint variable $\boldsymbol{w}$ is a solution of the adjoint system given by

$\frac{d \boldsymbol{w}^{T}}{d t}=-\boldsymbol{w}^{T} \boldsymbol{A}+\left(y-y_{t}\right)^{T} \boldsymbol{C}$,

or, taking transposes of all terms,

$\frac{d \boldsymbol{w}}{d t}=-\boldsymbol{A}^{T} \boldsymbol{w}+\boldsymbol{C}^{T}\left(y-y_{t}\right)$,

subject to the terminal condition $\boldsymbol{w}\left(t_{f}\right)=0$. The adjoint system is solved backwards in time, starting from the terminal condition $\boldsymbol{w}\left(t_{f}\right)=0$ in order to obtain $\boldsymbol{w}(t)$.

Finally, the variation of $\mathcal{L}$ with respect to the input $u$ provides the sensitivity to drug application. It is given by

$\delta \mathcal{L}_{\delta u}=-\int_{0}^{t_{f}} \boldsymbol{w}^{T} \boldsymbol{B} \delta u d t$,

and thus the changes in input leading to the highest decrease in $\mathcal{L}$ are

$\delta u=\boldsymbol{B}^{T} \boldsymbol{w}$. 


\section{Optimization algorithm}

The sensitivities are used in an optimal-control algorithm to find the drug administration that minimizes $\mathcal{L}$. We start with zero drug administration. This first input is referred to as $u_{1}(t)$. We then set an iterative process, where the variation of $\mathcal{L}$ with respect to the dose $u$ is calculated at each iteration, and an updated guess of the optimal dose is obtained with Eq. (22) in iterations $i=1,2,3, \ldots$. The steps for iteration $i$ are:

1. Solve the direct PK system given by Eq. (9), with $u(t)=u_{i}(t)$. This provides the direct solution of iteration $i, \boldsymbol{x}_{i}(t)$, and the corresponding output $y_{i}(t)$ is obtained from Eq. (11);

2. Solve the adjoint PK system given by Eq. (20). This provide the adjoint solution of iteration $i, \boldsymbol{w}_{i}(t)$;

3. The change in drug dosage leading to the optimal decrease in the functional $\mathcal{L}, \delta u_{i}$, is given by Eq. (22), and the dose for iteration $i+1$ is updated as $u_{i+1}=u_{i}+\epsilon \delta u_{i}$, with $\epsilon$ given as a small constant to ensure small dose updates.

The steps above are shown in schematic form in Fig. 2. Successive iterations are carried out until $\delta u$ vanishes. When this happens the variations of $\mathcal{L}$ with respect to $\boldsymbol{x}, \boldsymbol{w}$ and $u$ are simultaneously zero, and we have thus reached a minimum.

\section{Constraints in drug administration}

The procedure of the previous section leads to a continuous profile for drug administration, $u(t)$, which is optimal to attain the target concentration but is impractical for application, as it requires a continuous infusion with variable rate. In clinical practice, each infusion is applied with a constant rate over a period of time, which for colistin could be one hour $[18,40]$. This amounts to a restriction to $u(t)$, which should be constant during an infusion and zero otherwise. An infusion rate satisfying such constraint may be obtained from the optimal $\delta u(t)$ as

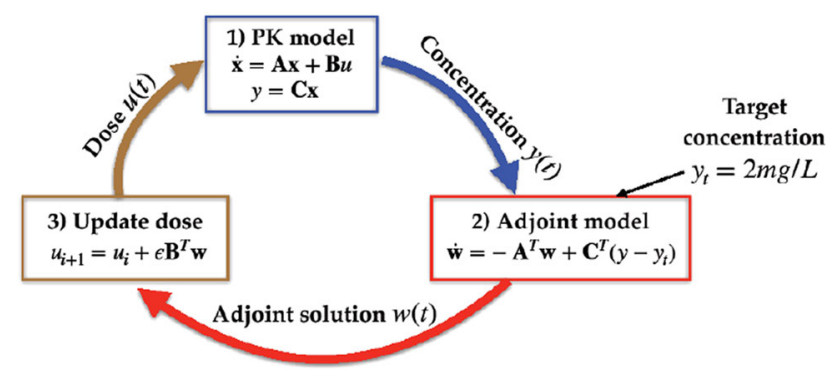

Fig. 2 Schematic representation of the optimization process $\delta u_{m o d}(t)=\left\{\begin{array}{cc}\overline{\delta u} & \text { if } t \text { is within an infusion period } \\ 0 & \text { otherwise }\end{array}\right.$

where the overbar denotes a time average within an infusion period. Hence, changes to the infusion rate $u$ are constant in each specified infusion period, with a value equal to the time average of the optimal change within each period, and are zero for times between infusions. This ensures that the resulting optimization of drug administration satisfy the practical constraint of constant infusion rate within a fixed period. In this study, we have used a fixed infusion period of one hour, and tested different intervals between infusions. Periods of 0.5 to $1 \mathrm{~h}$ have been recommended in the literature [26]. If necessary, changing the infusion time in the optimization is straightforward. Some tests were carried out with a $2 \mathrm{~h}$ infusion period, leading to minimal differences to the reported optimal doses for $1 \mathrm{~h}$ infusions.

\section{Extensions}

The above procedure allows extensions for other optimization scenarios of interest, which are described in what follows.

\section{Inter-individual variability}

The optimal control problem was here formulated for a deterministic model, with $\boldsymbol{A}, \boldsymbol{B}$ and $\boldsymbol{C}$ matrices held fixed by considering median values of pharmacokinetic parameters. Nonetheless, the optimization may be extended by considering a population analysis. A straightforward approach is the use of a Monte Carlo method to generate a population of patients with varying pharmacokinetic parameters (clearances and volumes of distribution), which in the framework leads to separate $\boldsymbol{A}_{\boldsymbol{i}}, \boldsymbol{B}_{\boldsymbol{i}}$ and $\boldsymbol{C}_{\boldsymbol{i}}$ matrices for each patient $i$. In this case, an appropriate objective functional is

$\mathcal{J}_{P O P}=\frac{1}{2} \sum_{i} \int_{0}^{t_{f}}\left[y_{i}(t)-y_{t}(t)\right]^{2} d t$

which sums deviations of the concentration to the target value for each patient. With this choice, the sensitivity of $\mathcal{J}_{\text {POP }}$ to changes in the dose is simply the sum of the sensitivity to each patient. Simulations of the direct and adjoint PK systems, considering matrices $\boldsymbol{A}_{\boldsymbol{i}}, \boldsymbol{B}_{\boldsymbol{i}}$ and $\boldsymbol{C}_{\boldsymbol{i}}$, provide such sensitivities and allow updating the dose in the optimization loop. The optimized dose so obtained minimizes the objection in Eq. (24), thus leading to the dose that leads to the lowest deviations of concentration from its target value once averaged among the considered individuals. 
In this case, an interesting possibility is the inclusion of toxicity considerations. The objective functional of Eq. (24) may be supplemented with penalization terms related to patients that reach toxic concentrations, similar to Pierillas et al. [41]. Such penalization terms appear as costs introduced to the objective functional, as in Kirschner et al. [30] and Joshi [29]. The introduction of penalization terms to the objective functional and subsequent derivation of the optimal control problem should reduce toxicity cases. Notice however that the use of a target concentration of $2 \mathrm{mg} / \mathrm{L}$ in the present framework already limits toxicity, as the optimization naturally converges to this concentration avoiding values significantly above the target.

\section{Nonlinear models}

The optimal control problem was formulated for a linear PK model due to its widespread use for most drugs [28], and, in particular, to colistin [22], which is the subject of this work. This provides a general framework that may be applied for any linear PK model, once written in the form of Eq. (9). Optimal control can also be applied to nonlinear models, such as in the works of Kirschner et al. [30] and Joshi [29] on optimal control of HIV chemotherapy or Sharp et al. [35] on acute myeloid leukaemia. The framework is the same, with the definition of an augmented functional $\mathcal{L}$. The adjoint equation is no longer Eq. (20), and needs to be derived for the specific nonlinear model at hand. Once derived and solved, the adjoint solution provides the sensitivity to drug administration.

\section{Concentration at a different compartment}

In this work we have considered a target colistin plasma concentration of $2 \mathrm{mg} / \mathrm{L}$, as previous works [25, 26]. For models including an ELF compartment, such as Boisson et al. [18], it is possible to specify a target concentration in the ELF by adopting the appropriate output matrix $\boldsymbol{C}$. More generally, the selection of output compartment and target concentration in a given PK model is arbitrary, and allows dose optimization for any desired concentration profile.

\section{Numerical methods}

The procedure outlined in this section was implemented in a MATLAB script. Simulations of the direct and adjoint linear systems were carried out using the "lsim" routine. Time was discretized in intervals of $0.1 \mathrm{~h}$, and reductions of this value did not lead to significant changes in the results. We have checked the implementation of the PK model by simulating cases from [22], with results consistent to the published ones. The model parameters are reported in Table 1 . The small constant $\epsilon$ to adjust the drug administration was chosen to ensure small changes in $\mathcal{L}$ at each iteration. The process was stopped when relative changes to $\mathcal{L}$ were lower than $10^{-4}$.

\section{Results}

\section{Optimal dosage for a patient with normal renal function}

As defined in the previous section, lower values of $\gamma$ indicate a lower standard deviation between the total (bound plus unbound) colistin concentration and the target value. Therefore, the lower the $\gamma$ value, the closer the colistin plasma concentration will be to the target, which was taken here as $y_{t}(t)=2 \mathrm{mg} / \mathrm{L}[25,26]$.

Figure 2 shows the results of dosage optimization, considering patients with normal renal function (creatinine clearance $=80 \mathrm{~mL} / \mathrm{min}$ ), with different infusion regimens. The lowest determined value of $\gamma$ was $0.16 \mathrm{mg} / \mathrm{L}$ (Fig. 3b) for a dosage regimen with an infusion every $8 \mathrm{~h}$. For infusions every 12 and $24 \mathrm{~h}$, the values of $\gamma$ were higher: $0.19 \mathrm{mg} / \mathrm{L}$ (every $12 \mathrm{~h}$ ) and $0.36 \mathrm{mg} / \mathrm{L}$ (every $24 \mathrm{~h}$ ), respectively (Fig. 3d and f). An administration every $6 \mathrm{~h}$ for a patient with normal renal function leads to a value of $\gamma$ equal to $0.15 \mathrm{mg} / \mathrm{L}$ (data not shown), a marginal improvement compared to the value determined for a regimen every $8 \mathrm{~h}$.

The optimized IV administration of CMS in different dosing regimens (Fig. 3a, c and e) resulted in profiles of CMS and colistin plasma concentrations as a function of the time shown in Fig. 3b, d and f.

For a treatment every $8 \mathrm{~h}$, the optimal control is a loading dose of approximately 8 million international units (MIU) of CMS (equivalent to about $640 \mathrm{mg}$ of CMS and $264 \mathrm{mg}$ of colistin base activity (CBA)), followed by approximately $0.5 \mathrm{MIU}$ of CMS after $8 \mathrm{~h}$ and a maintenance dose, starting after $16 \mathrm{~h}$, of approximately $2.4 \mathrm{MIU}$ of CMS, totalizing 7.2 MIU daily of CMS (Fig. 3a).

For a regimen with administration every $12 \mathrm{~h}$, the optimized loading doses were around 8 MIU of CMS followed by $2.5 \mathrm{MIU}$ after $12 \mathrm{~h}$ and the maintenance dose was around 3.6 MIU of CMS (7.2 MIU daily of CMS) (Fig. 3c). For a regimen with infusion every $24 \mathrm{~h}$, the optimal loading dose was around $9 \mathrm{MIU}$ and the maintenance dose was around 6.8 MIU (Fig. 3e). In the regimens with more frequent infusions, the first doses obtained from the optimization procedure form a loading profile, with a first dose that quickly leads to the target value, followed by a second, lower dose that maintains the concentration around the target 
Fig. 3 Optimal profile for a patient with normal renal function. Dose optimization for patients with normal renal function (creatinine clearance $=80 \mathrm{~mL} / \mathrm{min})$ after an administration every $8 \mathrm{~h}$ (a), $12 \mathrm{~h} \mathrm{(c)}$ and $24 \mathrm{~h}(\mathbf{e})$. CMS in the central (dotted line) and peripheral (light gray line) compartments and colistin plasma concentrations (dark gray line) over time for a regimen with administration every $8 \mathrm{~h} \mathrm{(b)} 12 \mathrm{~h}$ (d) and $24 \mathrm{~h}$ (f). $\gamma$ : Standard deviation from target concentration $2 \mathrm{mg} / \mathrm{L}$; CMS: colistimethate sodium; MIU: million international units (a) Every $8 \mathrm{~h}$

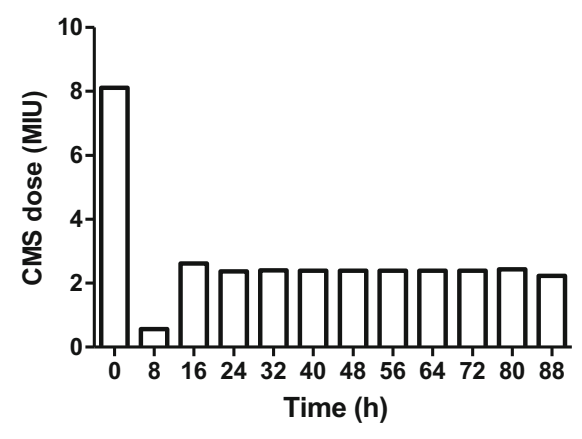

(c) Every $12 \mathrm{~h}$

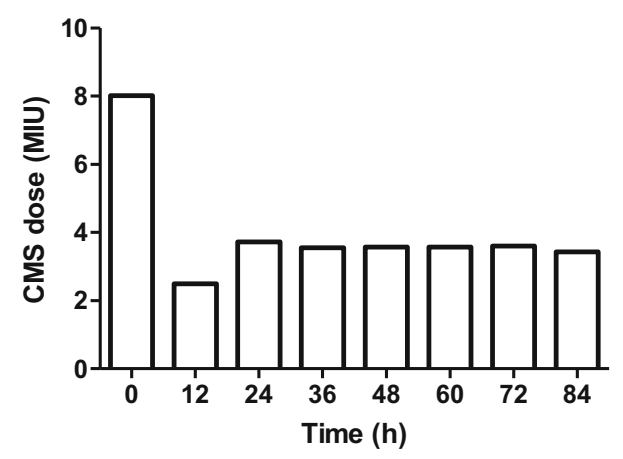

(e) Every $24 \mathrm{~h}$

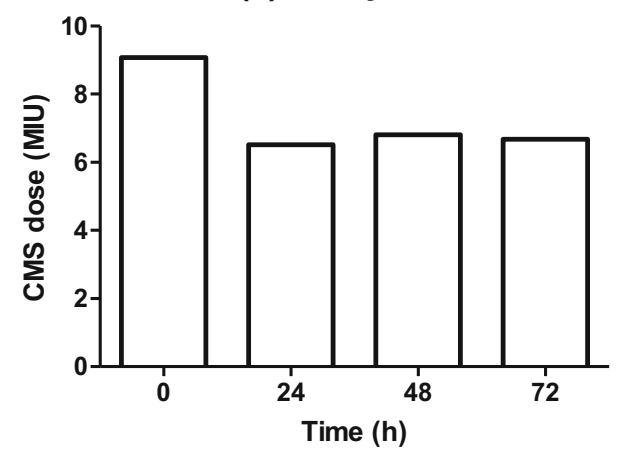

(b) $\gamma=0.16 \mathrm{mg} / \mathrm{L}$

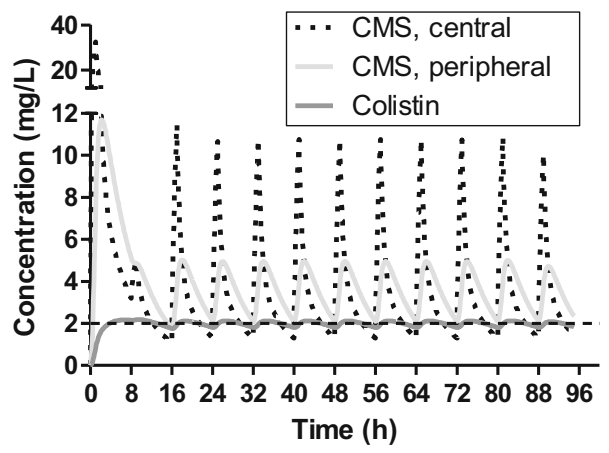

(d) $\gamma=0.19 \mathrm{mg} / \mathrm{L}$

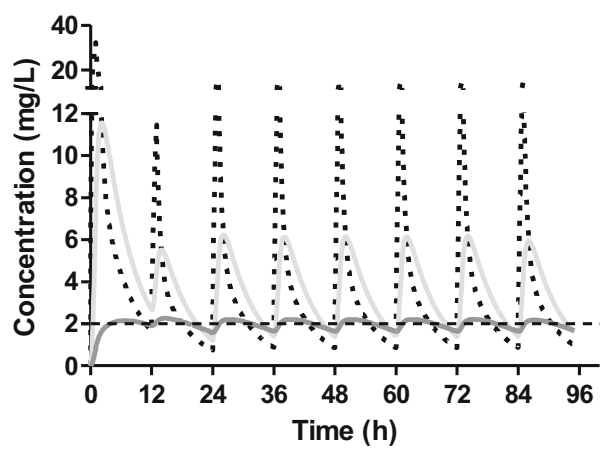

(f) $\gamma=0.36 \mathrm{mg} / \mathrm{L}$

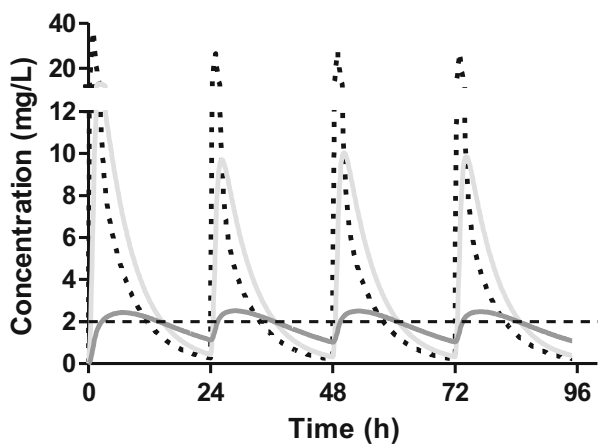

\section{Optimal dosage regimen of colistin for patients with different renal conditions}

As the PK model is a function of creatinine clearance, separate optimizations should be carried out depending on the renal function. Thus, the optimal dose varies (Fig. 4a, c and e) with the renal function to maintain the colistin plasma concentrations close to the target value of $2 \mathrm{mg} / \mathrm{mL}$ (Fig. 4b, d and f). For a patient with creatinine clearance equal to $20 \mathrm{~mL} / \mathrm{min}$, the doses need to be reduced, and the slower absorption of colistin leads to more complex optimal loading dose profiles; for an infusion every $8 \mathrm{~h}$, the optimal loading profile of CMS in the first $24 \mathrm{~h}$ were about 4, $0,0.125$ and $1 \mathrm{MIU}$ at times $0,8,16$ and $24 \mathrm{~h}$, respectively. In this case the optimal loading profile involves four initial administrations, so as to attain quickly the target concentration with a high initial dose (Fig. 4a and $b$ ), and subsequently maintain the concentration with zero or low doses. The optimal maintenance dose was around 0.8 MIU of CMS every $8 \mathrm{~h}$ (Fig. 4a). For infusion every $12 \mathrm{~h}$, the optimal loading profile of CMS is of 4.5, 0 and 1.4 MIU at times 0, 12 and $24 \mathrm{~h}$, respectively (Fig. 4c). For infusions occurring every $24 \mathrm{~h}$, the optimal loading profile is of 4.4 and $2 \mathrm{MIU}$ at times 0 and $24 \mathrm{~h}$, respectively (Fig. 4e). The optimal maintenance doses were 1.2 and 2.4 MIU for infusions every 12 and $24 \mathrm{~h}$, respectively (Figs. 4c and e). The optimal values of $\gamma$ for administrations every 8 , 12 or $24 \mathrm{~h}$ were, respectively, $0.18,0.19$ and $0.23 \mathrm{mg} / \mathrm{L}$ for 
Fig. 4 Optimal profile for patients with different renal conditions. Dose optimization for patients with creatinine clearance equal to $20 \mathrm{~mL} / \mathrm{min}$ (black bar) $80 \mathrm{~mL} / \mathrm{min}$ (white bar) and $150 \mathrm{~mL} / \mathrm{min}$ (gray bar) after an administration every $8 \mathrm{~h}(\mathbf{a}), 12 \mathrm{~h}(\mathbf{c})$ and $24 \mathrm{~h} \mathrm{(e).}$ Colistin plasma concentrations over time for patients with creatinine clearance equal to $20 \mathrm{~mL} / \mathrm{min}$ (dotted line) $80 \mathrm{~mL} /$ min (light gray line) and $150 \mathrm{~mL} / \mathrm{min}$ (black line) for a regimen with administrations every $8 \mathrm{~h}(\mathbf{b}) 12 \mathrm{~h} \mathrm{(d)}$ and $24 \mathrm{~h}$ (f). CrCL: creatinine clearance; $\gamma$ : Standard deviation from target concentration $2 \mathrm{mg} / \mathrm{L}$; CMS: colistimethate sodium; MIU: million international units
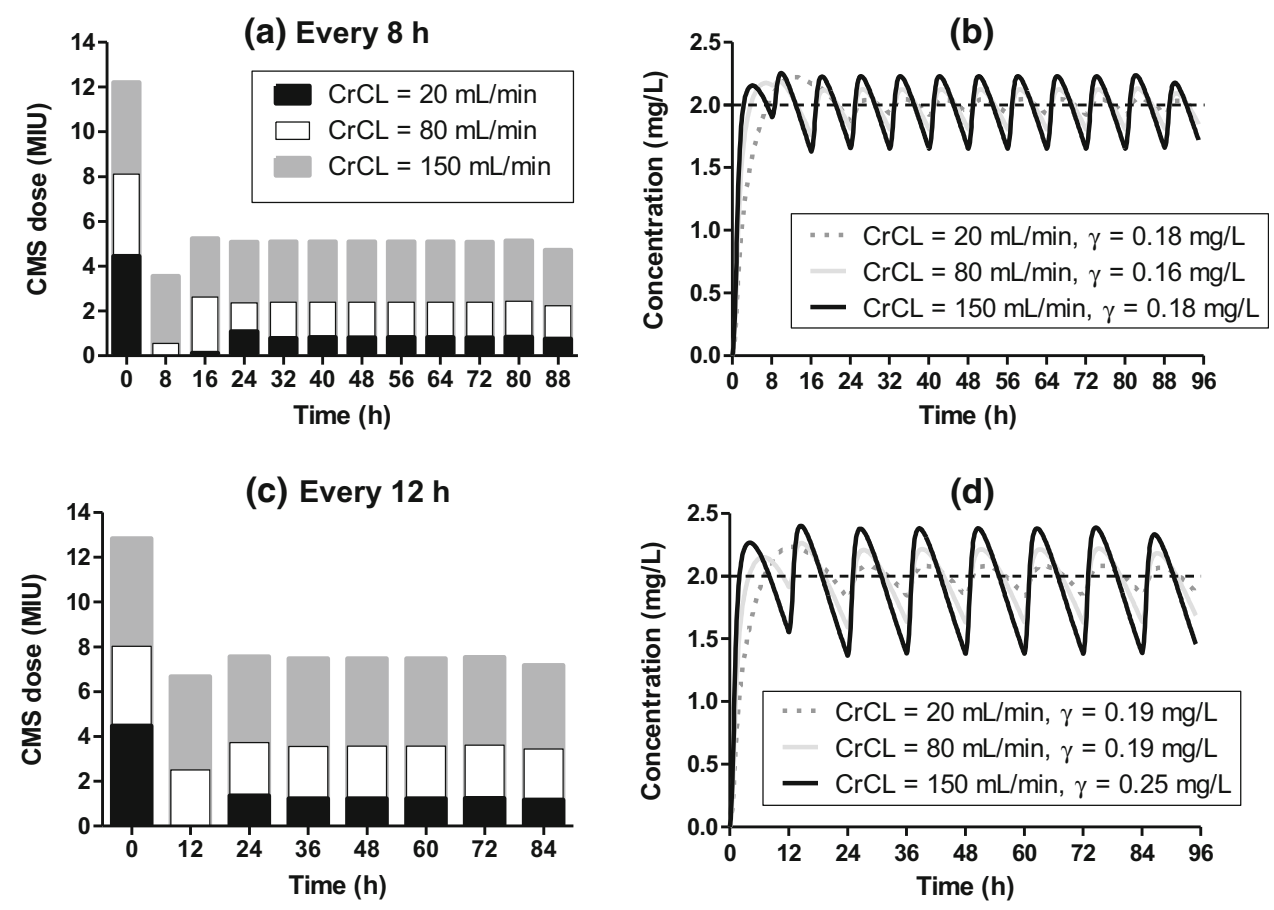

(d)

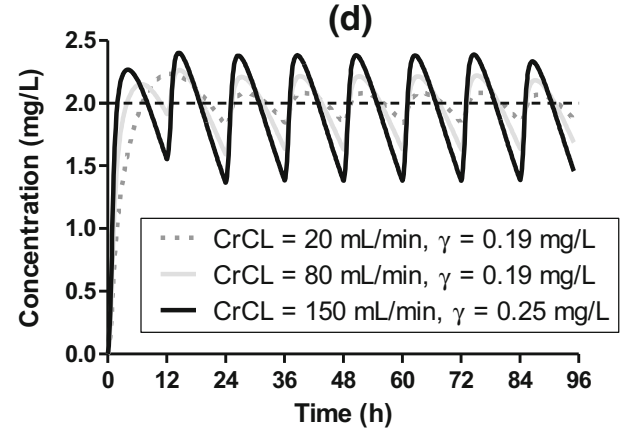

(e) Every $24 \mathrm{~h}$

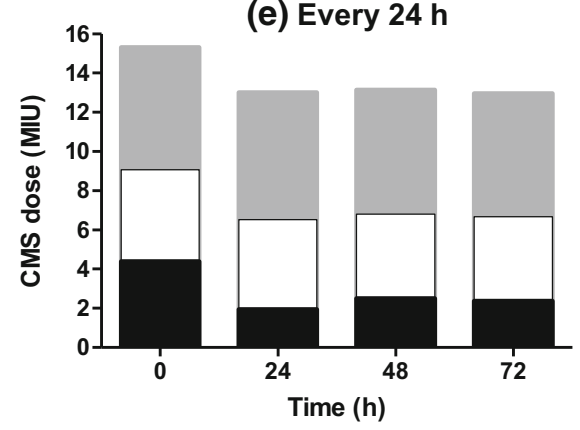

(f)

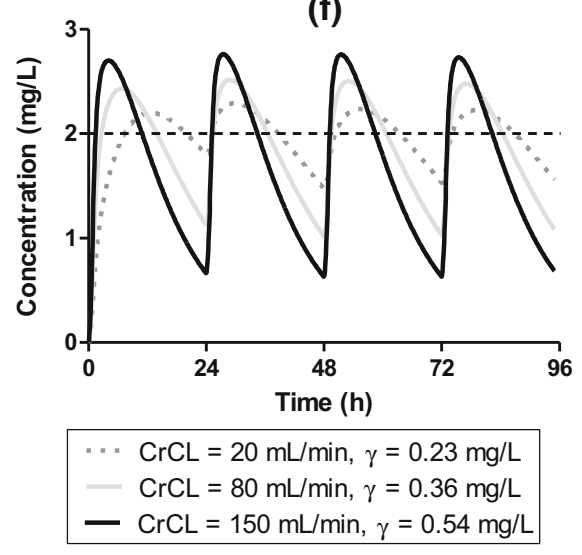

a patient with creatinine clearance equal to $20 \mathrm{~mL} / \mathrm{min}$ (Figs. 4b, d and f).

For patients presenting creatinine clearance equal to $150 \mathrm{~mL} / \mathrm{min}$, the optimal dose is higher as shown in the Figs. $4 \mathrm{a}$, c and e. With infusions every $8 \mathrm{~h}$, the first two doses obtained in the optimization are about 12 and 3.5 MIU, followed by a maintenance dose of 5 MIU. Infusions every $12 \mathrm{~h}$ lead to initial doses of 13 and 6.5 MIU and a maintenance dose of 7.5 MIU, and for infusions every $24 \mathrm{~h}$ the optimization leads to a single loading dose of about 15.5 MIU followed by a maintenance dose of 13 MIU. The values of $\gamma$ for administrations every 8,12 or $24 \mathrm{~h}$ were $0.18,0.25$ and $0.54 \mathrm{mg} / \mathrm{L}$, respectively (Figs. $4 \mathrm{~b}$, d and f).

\section{Discussion}

This study used control theory to optimize drug administration. Colistin was chosen for the analysis since: (i) its ideal dosage regimen is not fully established yet; (ii) it is often used in the clinical practice and is frequently the only effective option for the treatment of certain infections; (iii) it possesses a narrow therapeutic window and should be administered carefully in order to avoid toxicity issues.

The target plasma concentration of colistin $(2 \mathrm{mg} / \mathrm{L})$ was selected since this concentration seems to be effective against serious infectious diseases by Gram-negative bacteria $[25,26]$. Moreover, a previous study showed that plasma colistin concentrations greater than $2.42 \mathrm{mg} / \mathrm{L}$ were more related to nephrotoxicity [27]. Therefore, this target seems to be a satisfactory balance between effectiveness 
and toxicity. The optimization procedure leads to doses that minimize the standard deviation $\gamma$ between colistin plasma concentration and its target value.

In the optimal control results, the IV administration every $8 \mathrm{~h}$ led to a lower $\gamma$ value $(0.16 \mathrm{mg} / \mathrm{L})$ than for every 12 or $24 \mathrm{~h}(0.19$ and $0.36 \mathrm{mg} / \mathrm{L}$, respectively $)$. The difference is less substantial between administrations every 8 and $12 \mathrm{~h}$, but the values are considerably lower than what is obtained with an administration every $24 \mathrm{~h}$, suggesting that a shorter interval between the infusions could favor the success of the treatment with colistin, as a more frequent administration leads to lower fluctuations of the concentrations, keeping the values close to the target plasma concentration (Fig. 3b, d and f). Additionally, a shorter interval between administrations decreases the possibility of developing resistance to colistin [42, 43].

Previous studies showed that in cases of lung infections caused by bacteria presenting high values of MIC for colistin, the target plasma concentration should be greater than $2 \mathrm{mg} / \mathrm{L}[25,44,45]$. As the PK model used in this work is linear, optimal doses for other target concentrations may be obtained directly by multiplying the doses reported here by a factor. For instance, a target concentration of $3 \mathrm{mg} / \mathrm{L}$ is obtained by multiplying all doses by 1.5 , leading to a daily maintenance dose of about 10.5 MIU of CMS. The maintenance doses (around 7 MIU of CMS daily) suggested by this present model for a patient with a normal renal function are higher than the doses frequently used in the clinical practice (3 to 6 MIU of CMS per day) [21, 46]. However, this suggested dose is lower than the dose recently recommended by the International Consensus Guidelines for the Optimal Use of the Polymyxins for a patient presenting a creatinine clearance of $80 \mathrm{~mL} / \mathrm{min}$ (10.3 MIU of CMS) [26]. This could be explained by the fact that the latter consensus was based on works [22, 25] that developed a dosing algorithm that did not use the median values of obtained data. Instead of this, the authors performed dosing adjustments such that more than $80 \%$ of the patients achieve concentrations equal or higher than the target. We have used the same model [22], but we have used the median values, which means that $50 \%$ of the patients would be above and the other $50 \%$ would be below the target concentration.

The recommended loading dose could vary between 6 to 9 MIU of CMS with the subsequent first maintenance dose starting after 8,12 or $24 \mathrm{~h}$ [10, 26]. Interestingly, the loading dose obtained as optimal control was different from those often used by physicians. The optimal loading regimen is divided into 2 or more administrations, especially for shorter intervals between the administrations. A high loading dose is initially recommended followed by a dose much lower than the first one. This regimen is interesting for the treatment with colistin, since it allows one to attain more rapidly the target plasma concentration, avoiding plasma concentrations much higher than the target. Such loading regimen deserves further investigation.

One of the advantages of the present method is that it allows the selection of doses for specific patients. As most of CMS is excreted in the urine, it is expected that a reduction in the renal function increases the plasma concentration of CMS and, consequently, that of colistin. This requires a dose reduction, as demonstrated in previous works [22, 25], and also obtained in this study. Interestingly, for a patient presenting creatinine clearance equal to $20 \mathrm{~mL} / \mathrm{min}$ the $\gamma$ values after an administration every 8,12 and $24 \mathrm{~h}$ were comparable $(0.18,0.19$ and $0.23 \mathrm{mg} / \mathrm{L})$ (Figs. 4b, d and f). This can be explained by the fact that this patient eliminates CMS more slowly, which results in a lower fluctuation of the plasma concentrations over time, keeping these concentrations closer to the target values, even after an administration every $24 \mathrm{~h}$. On the other hand, for a patient presenting high creatinine clearance, such as $150 \mathrm{~mL} / \mathrm{min}$, the $\gamma$ values were considerably lower for a shorter interval between the infusions $(0.18,0.25$ and $0.54 \mathrm{mg} / \mathrm{L}$ ) after an administration every 8, 12 and $24 \mathrm{~h}$, respectively, Figs. 4b, d and f). Such augmented renal clearance is often observed for patients with traumatic injury [2]. The faster elimination of CMS suggests a treatment with shorter intervals between infusions, which, when optimized, leads to lower deviations of colistin plasma concentration to its target value. For the larger interval of $24 \mathrm{~h}$ (Fig. 4f) the higher clearance leads to a sharp decrease of colistin concentration between infusions, with a significant period with concentrations lower than $2 \mathrm{mg} / \mathrm{L}$.

Here the focus was on the effect of creatinine clearance on the optimal doses, but it is straightforward to perform optimizations varying other parameters of the PK model. For instance, the model in [22] is a function of patient weight. In the present results we have considered a patient weighing $60 \mathrm{~kg}$. Separate optimizations may be performed to select appropriate doses for other chosen patient weights. Moreover, the model also includes parameters for patients undergoing hemodialysis or continuous renal replacement therapy, and inclusion of these in the model enables optimizations for such patients as well.

\section{Conclusion}

The present work presented a new method to optimize dose selection based on a PK model, using optimal control. Here, the framework is used to guide the choice of the optimal colistin dose after an IV administration of CMS. This method can be applied for new molecules and other clinically relevant drugs, with clearer benefits for cases 
with a narrow therapeutic window such as colistin, as the optimization minimizes the deviations between a model result (here, the colistin plasma concentration) and a target value that may be specified based on the available knowledge about the drug at hand. The optimized doses show differences to currently used values, especially for a loading regimen which often uses multiple administrations with varying doses, leading to a faster attainment of the target concentration without toxicity issues. Moreover, the quantification of the optimal standard deviation $\gamma$ allows an evaluation of potential benefits of lower administration intervals. Further studies with preclinical assays and clinical trials in humans would be relevant in order to confirm these results.

\section{Declarations}

Conflict of interest The authors have no relevant financial or nonfinancial interests to disclose. The authors have no conflicts of interest to declare that are relevant to the content of this article. All authors certify that they have no affiliations with or involvement in any organization or entity with any financial interest or non-financial interest in the subject matter or materials discussed in this manuscript. The authors have no financial or proprietary interests in any material discussed in this article.

\section{References}

1. Theuretzbacher U, Gottwalt S, Beyer P, Butler M, Czaplewski L, Lienhardt C, Moja L, Paul M, Paulin S, Rex JH, Silver LL, Spigelman M, Thwaites GE, Paccaud J-P, Harbarth S (2019) Analysis of the clinical antibacterial and antituberculosis pipeline. Lancet Infect Dis 19(2):e40-e50. https://doi.org/10.1016/ S1473-3099(18)30513-9

2. Sime FB, Roberts MS, Roberts JA (2015) Optimization of dosing regimens and dosing in special populations. Clin Microbiol Infect 21(10):886-893. https://doi.org/10.1016/j.cmi.2015.05.002

3. Mouton JW, Brown DFJ, Apfalter P, Cantón R, Giske CG, Ivanova M, MacGowan AP, Rodloff A, Soussy CJ, Steinbakk M, Kahlmeter G (2012) The role of pharmacokinetics/pharmacodynamics in setting clinical MIC breakpoints: the EUCAST approach. Clin Microbiol Infect 18(3):E37-E45. https://doi.org/ 10.1111/j.1469-0691.2011.03752.x

4. Nielsen EI, Cars O, Friberg LE (2011) Pharmacokinetic/pharmacodynamic (PK/PD) indices of antibiotics predicted by a semimechanistic PKPD model: a step toward model-based dose optimization. Antimicrob Agents Chemother 55(10):4619-4630

5. Rizk ML, Bhavnani SM, Drusano G, Dane A, Eakin AE, Guina T, Jang SH, Tomayko JF, Wang J, Zhuang L, Lodise TP (2019) Considerations for dose selection and clinical pharmacokinetics/ pharmacodynamics for the development of antibacterial agents. Antimicrob Agents Chemother 63(5):e02309-e02318. https://doi. org/10.1128/aac.02309-18

6. Roberts JA, Norris R, Paterson DL, Martin JH (2012) Therapeutic drug monitoring of antimicrobials. $\mathrm{Br} \mathrm{J}$ Clin Pharmacol 73(1):27-36. https://doi.org/10.1111/j.1365-2125.2011.04080.x

7. de Velde F, Mouton JW, de Winter BCM, van Gelder T, Koch BCP (2018) Clinical applications of population pharmacokinetic models of antibiotics: challenges and perspectives. Pharmacol Res 134:280-288

8. Jönsson S, Karlsson MO (2003) A rational approach for selection of optimal covariate-based dosing strategies. Clin Pharmacol Ther 73(1):7-19

9. Nielsen EI, Friberg LE (2013) Pharmacokinetic-pharmacodynamic modeling of antibacterial drugs. Pharmacol Rev 65(3):1053-1090

10. Mohamed AF, Karaiskos I, Plachouras D, Karvanen M, Pontikis K, Jansson B, Papadomichelakis E, Antoniadou A, Giamarellou $\mathrm{H}$, Armaganidis A (2012) Application of a loading dose of colistin methanesulfonate in critically ill patients: population pharmacokinetics, protein binding, and prediction of bacterial kill. Antimicrob Agents Chemother 56(8):4241-4249

11. Yao X, Ye F, Zhang M, Cui C, Huang B, Niu P, Liu X, Zhao L, Dong E, Song C (2020) In vitro antiviral activity and projection of optimized dosing design of hydroxychloroquine for the treatment of severe acute respiratory syndrome coronavirus 2 (SARSCoV-2). Clin Infect Dis 71(15):732-739

12. Evans ME, Feola DJ, Rapp RP (1999) Polymyxin B sulfate and colistin: old antibiotics for emerging multiresistant gram-negative bacteria. Ann Pharmacother 33(9):960-967

13. Gai Z, Samodelov SL, Kullak-Ublick GA, Visentin M (2019) Molecular mechanisms of colistin-induced nephrotoxicity. Molecules 24(3):653

14. Li J, Nation RL, Milne RW, Turnidge JD, Coulthard K (2005) Evaluation of colistin as an agent against multi-resistant Gramnegative bacteria. Int J Antimicrob Agents 25(1):11-25. https:// doi.org/10.1016/j.ijantimicag.2004.10.001

15. Paul M, Daikos GL, Durante-Mangoni E, Yahav D, Carmeli Y, Benattar YD, Skiada A, Andini R, Eliakim-Raz N, Nutman A (2018) Colistin alone versus colistin plus meropenem for treatment of severe infections caused by carbapenem-resistant Gramnegative bacteria: an open-label, randomised controlled trial. Lancet Infect Dis 18(4):391-400

16. Ehrmann S, Luyt C-E (2020) Optimizing aerosol delivery of antibiotics in ventilated patients. Current Opinion in Infectious Diseases 33(2): 197-204

17. Michalopoulos A, Kasiakou SK, Mastora Z, Rellos K, Kapaskelis AM, Falagas ME (2005) Aerosolized colistin for the treatment of nosocomial pneumonia due to multidrug-resistant Gram-negative bacteria in patients without cystic fibrosis. Crit Care 9(1):R53

18. Boisson M, Jacobs M, Grégoire N, Gobin P, Marchand S, Couet W, Mimoz O (2014) Comparison of intrapulmonary and systemic pharmacokinetics of colistin methanesulfonate (CMS) and colistin after aerosol delivery and intravenous administration of CMS in critically ill patients. Antimicrob Agents Chemother 58(12):7331-7339

19. Gontijo AVL, Grégoire N, Lamarche I, Gobin P, Couet W, Marchand S (2014) Biopharmaceutical characterization of nebulized antimicrobial agents in rats: 2. Colistin. Antimicrobial agents and chemotherapy 58(7):3950-3956

20. Couet W, Gregoire N, Gobin P, Saulnier PJ, Frasca D, Marchand S, Mimoz O (2011) Pharmacokinetics of colistin and colistimethate sodium after a single $80-\mathrm{mg}$ intravenous dose of CMS in young healthy volunteers. Clin Pharmacol Ther 89(6):875-879

21. Sorlí L, Luque S, Li J, Campillo N, Danés M, Montero M, Segura C, Grau S, Horcajada JP (2019) Colistin for the treatment of urinary tract infections caused by extremely drug-resistant Pseudomonas aeruginosa: dose is critical. J Infect 79(3):253-261

22. Garonzik SM, Li J, Thamlikitkul V, Paterson DL, Shoham S, Jacob J, Silveira FP, Forrest A, Nation RL (2011) Population pharmacokinetics of colistin methanesulfonate and formed colistin in critically ill patients from a multicenter study provide dosing suggestions for various categories of patients. Antimicrob Agents Chemother 55(7):3284-3294 
23. Jacobs M, Gregoire N, Megarbane B, Gobin P, Balayn D, Marchand S, Mimoz O, Couet W (2016) Population pharmacokinetics of colistin methanesulfonate and colistin in critically ill patients with acute renal failure requiring intermittent hemodialysis. Antimicrob Agents Chemother 60(3):1788-1793

24. Bellos I, Pergialiotis V, Frountzas M, Kontzoglou K, Daskalakis G, Perrea DN (2020) Efficacy and safety of colistin loading dose: a meta-analysis. J Antimicrob Chemotherapy. 75(10):2725-2734

25. Nation RL, Garonzik SM, Thamlikitkul V, Giamarellos-Bourboulis EJ, Forrest A, Paterson DL, Li J, Silveira FP (2017) dosing guidance for intravenous colistin in critically Ill patients. Clin Infect Dis 64(5):565-571. https://doi.org/10.1093/cid/ciw839

26. Tsuji BT, Pogue JM, Zavascki AP, Paul M, Daikos GL, Forrest A, Giacobbe DR, Viscoli C, Giamarellou H, Karaiskos I (2019) International Consensus Guidelines for the Optimal Use of the Polymyxins: Endorsed by the American College of Clinical Pharmacy (ACCP), European Society of Clinical Microbiology and Infectious Diseases (ESCMID), Infectious Diseases Society of America (IDSA), International Society for Anti-infective Pharmacology (ISAP), Society of Critical Care Medicine (SCCM), and Society of Infectious Diseases Pharmacists (SIDP). Pharmacotherapy 39(1):10-39

27. Sorlí L, Luque S, Grau S, Berenguer N, Segura C, Montero MM, Álvarez-Lerma F, Knobel H, Benito N, Horcajada JP (2013) Trough colistin plasma level is an independent risk factor for nephrotoxicity: a prospective observational cohort study. BMC Infect Dis 13(1):380. https://doi.org/10.1186/1471-2334-13-380

28. Derendorf H, Schmidt S (2019) Rowland and Tozer's clinical pharmacokinetics and pharmacodynamics: concepts and applications, 5th edn. Lippincott Williams \& Wilkins, Philadelphia

29. Joshi HR (2002) Optimal control of an HIV immunology model. Opt Control Appl Methods 23(4):199-213

30. Kirschner D, Lenhart S, Serbin S (1997) Optimal control of the chemotherapy of HIV. J Math Biol 35(7):775-792

31. Angaroni F, Graudenzi A, Rossignolo M, Maspero D, Calarco T, Piazza R, Montangero S, Antoniotti M (2020) An optimal control framework for the automated design of personalized cancer treatments. Front Bioeng Biotechnol. https://doi.org/10.3389/ fbioe. 2020.00523

32. Ledzewicz U, Maurer H, Schättler H (2019) Optimal combined radio- and anti-angiogenic cancer therapy. J Optim Theory Appl 180(1):321-340. https://doi.org/10.1007/s10957-018-1426-y

33. Ledzewicz U, Schättler H (2006) Drug resistance in cancer chemotherapy as an optimal control problem. Discrete Contin Dyn Syst B 6(1):129

34. Sharp JA, Browning AP, Mapder T, Baker CM, Burrage K, Simpson MJ (2020) Designing combination therapies using multiple optimal controls. J Theor Biol 25:
35. Sharp JA, Browning AP, Mapder T, Burrage K, Simpson MJ (2019) Optimal control of acute myeloid leukaemia. J Theor Biol 470:30-42

36. Neilan RLM, Schaefer E, Gaff H, Fister KR, Lenhart S (2010) Modeling optimal intervention strategies for cholera. Bull Math Biol 72(8):2004-2018

37. Miyaoka TY, Lenhart S, Meyer JFCA (2019) Optimal control of vaccination in a vector-borne reaction-diffusion model applied to zika virus. J Math Biol 79(3):1077-1104

38. Choi W, Shim E (2020) Optimal strategies for social distancing and testing to control COVID-19. J Theor Biol 512:

39. Pontryagin LS, Boltyanskii VG, Gamkrelidze RV, Mishchenko YEF (1962) Mathematical theory of optimal processes. Wiley Interscience, New York

40. Grégoire N, Mimoz O, Mégarbane B, Comets E, Chatelier D, Lasocki S, Gauzit R, Balayn D, Gobin P, Marchand S (2014) New colistin population pharmacokinetic data in critically ill patients suggesting an alternative loading dose rationale. Antimicrob Agents Chemother 58(12):7324-7330

41. Pierrillas PB, Fouliard S, Chenel M, Hooker AC, Friberg LF, Karlsson MO (2018) Model-based adaptive optimal design (MBAOD) improves combination dose finding designs: an example in oncology. The AAPS journal 20(2):1-11

42. Bergen PJ, Li J, Nation RL, Turnidge JD, Coulthard K, Milne RW (2008) Comparison of once-, twice-and thrice-daily dosing of colistin on antibacterial effect and emergence of resistance: studies with Pseudomonas aeruginosa in an in vitro pharmacodynamic model. J Antimicrob Chemother 61(3):636-642

43. Michalopoulos AS, Falagas ME (2011) Colistin: recent data on pharmacodynamics properties and clinical efficacy in critically ill patients. Ann Intens Care 1(1):30

44. Cheah S-E, Wang J, Nguyen VTT, Turnidge JD, Li J, Nation RL (2015) New pharmacokinetic/pharmacodynamic studies of systemically administered colistin against Pseudomonas aeruginosa and Acinetobacter baumannii in mouse thigh and lung infection models: smaller response in lung infection. J Antimicrob Chemother 70(12):3291-3297. https://doi.org/10.1093/jac/dkv267

45. Nation RL, Garonzik SM, Li J, Thamlikitkul V, GiamarellosBourboulis EJ, Paterson DL, Turnidge JD, Forrest A, Silveira FP (2016) Updated US and European dose recommendations for intravenous colistin: how do they perform? Clin Infect Dis 62(5):552-558. https://doi.org/10.1093/cid/civ964

46. Grégoire N, Aranzana-Climent V, Magréault S, Marchand S, Couet W (2017) Clinical pharmacokinetics and pharmacodynamics of colistin. Clin Pharmacokinet 56(12):1441-1460

Publisher's Note Springer Nature remains neutral with regard to jurisdictional claims in published maps and institutional affiliations. 